\title{
Beyond Conceptual Inventories: Lessons From a Large-Scale
}

\section{Assessment ${ }^{*}$}

\author{
Beth Thacker, Ganesh Chapagain, Vanalet Rusuriye, Keith West \\ Texas Tech University, Lubbock, USA
}

\begin{abstract}
We report on the free-response (FR) component of a large-scale assessment at a large university. It has become common to use conceptual inventories not just for the assessment of students' understanding for research purposes, but for the assessment of courses, curricula, and pedagogies. However, it can be argued that these instruments are insufficient. They are not comprehensive and are limited in their assessment of skills beyond conceptual understanding, such as mathematical and laboratory skills, thinking skills, and explanatory ability. In large classes, these skills often go unassessed, partially due to the predominance of multiple-choice (MC) assessment. The MC assessment format limits an instructor's ability to assess more complex problem solving skills, thought processes, students' explanatory abilities and writing skills. This raises the question as to whether or not, students even develop these skills, if they are not assessed. We explored this and the related issues through the administration of a set of FR pre- and post- tests across all of our introductory courses, both calculus- and algebra- based, as part of a large-scale assessment project over a number of years. The project was designed to: (1) assess the introduction of research-based materials into the labs and recitations; and (2) assess the different pedagogical methods used in the lectures and labs. In this paper, we report on the results of the FR pre- and post- testing in the context of electricity and magnetism. We present both quantitative and qualitative results from the FR assessment. The results reveal a more comprehensive picture of our students' skills and abilities than from conceptual inventories alone, providing, in particular, information on thinking skills, laboratory skills, math skills, and explanatory ability not available from conceptual inventories and emphasizing the advantages of the use of multiple forms of assessment in the comparison of different pedagogies and in course and program assessment. We give examples of the types of problems administered, the skills assessed, the analysis of the solutions qualitatively and quantitatively, the results, and directions for future research.
\end{abstract}

Keywords: large-scale assessment, inquiry, thinking skills, program assessment

\footnotetext{
*Acknowledgements: Thanks for the National Institutes of Health (NIH) Challenge Grant (No. \#5RC1GM090897-02), "An Assessment of Multimodal Physics Lab Intervention Efficacy in STEM Education," to assess for interventions to the laboratory curriculum at Texas Tech University, PI's Beth Thacker and Kelvin Cheng.

We also thank Hani Dulli for his contribution to writing the pre- and post- test problems and the NIH for their support of this project, for the funding of NIH Challenge grant \#1RC1GM090897-02. Any opinions, findings and conclusions or recommendations expressed are those of the authors and do not necessarily reflect the views of the NIH.

Beth Thacker, Dr., associate professor, Physics Department, Texas Tech University.

Ganesh Chapagain, Project Manager, Texas Tech University.

Vanalet Rusuriye, Physics Teacher, Texas Tech University.

Keith West, Dr., Undergraduate Teaching Laboratory Director, Physics Department, Texas Tech University.
} 


\section{Introduction}

This paper reports on one element of a large-scale assessment project performed in the Physics Department at Texas Tech University (TTU) from 2009 to 2012. In Fall 2009, we awarded a National Institutes of Health (NIH) Challenge grant to assess the changes made in our laboratories and newly formed recitation sections in the introductory courses. We had introduced evidence-based (EB) materials into the labs and recitation sections of all of our introductory courses, calculus- and algebra- based. With the grant, we set out to assess the results of the implementations using a number of assessment instruments, including conceptual inventories (Halloun \& Hestenes, 1985; Ding, Chabay, Sherwood, \& Beichner, 2006; Maloney, O'Kuma, Hieggelke, \& Van, 2001; Hestenes \& Wells, 1992), locally written free-response (FR) pre- and post- tests, scientific attitude and scientific reasoning inventories (Adams, Perkins, Podolefsky, Dubson, Finkelstein, \& Wieman, 2006), and teaching assistant (TA) evaluation inventories (Lawson, 1978). It was a large-scale assessment of size and extent not usually carried out in large physics departments.

In a previous paper (MacIsaac \& Falconer, 2002), we described the departmental setting, instructional approaches, and some resistance to reform, including a lack of concurrence among faculty on pedagogical issues in lecture, lab, and recitation. However, because there was some dissension, we had a situation where we could study changes in the laboratories and recitation sections with different instructional approaches in the lecture. We could assess the impact of introducing EB reforms in the labs and recitations with and without EB instruction in the lectures. We also reported on the results of assessment by conceptual inventory in that paper.

We found from the conceptual inventory assessment that when EB materials were introduced in the labs and recitations, independent of the lecture pedagogy, there was an increase in students' conceptual inventory gains and there was also an increase in the results on conceptual inventories, if EB instruction was employed in the lecture. The highest conceptual inventory gains were achieved by the combination of EB lectures and laboratories in large class settings and by a hands-on, laboratory-based, inquiry-based (INQ) course (Thacker, Diaz, \& Eligon, 2007; Wilhelm, Thacker, \& Wilhelm, 2007) taught in a small class setting.

However, conceptual inventories, while valid, reliable, and relatively easy to analyze due to a fact that the multiple-choice (MC) format are not comprehensive, are usually focused only on a subset of concepts or skills, and are limited in their ability to assess problem-solving and thinking skills. FR format questions, on the other hand, are not limited to assessment of concepts, allow for the demonstration of thinking skills, decrease guessing and allow for more complex answers. They are, however, more time intensive to grade or evaluate. We show a comparison of the two formats in Table 1. Because the conceptual inventories do have drawbacks, we wanted to assess the students' performance on FR pre- and post- tests in addition to the assessment by conceptual inventories. The FR format allowed us to ask questions requiring evidence of complex thinking skills and to assess skills beyond conceptual understanding, such as laboratory skills, mathematical skills, explanatory ability, and writing skills.

In this paper, we report on the FR pre- and post- testing as part of the large-scale assessment. We give examples of the types of problems administered; discuss the skills assessed, the quantitative and qualitative analysis, the results, and directions for future research. Because we have a large amount of data, we limit our report in this paper to a few examples from FR pre- and post- testing in the recitation sections of the second semester (electricity and magnetism) of our large lecture courses in our introductory sequences. 
We discuss, in Section II, the department and student populations; in Section III, the development and administration of the assessment instruments; in Section IV, the analysis methods; in Section V, the results; and in Section VI, discussion and conclusions.

Table 1

Comparison of Assessment by Conceptual Inventory and FR Pre- and Post- Testing

\begin{tabular}{|l|l|}
\hline Conceptual inventories & FR pre- and post- testing \\
\hline Valid and reliable. & Allow for more complex answers. \\
Easy to score. & Allow for a demonstration of thinking skills. \\
Designed for research—not meant to be used as the sole & Not limited to the assessment of concepts. \\
assessment of a course or curriculum. & \\
Not comprehensive. & Likelihood of guessing is decreased. \\
Focused on a particular subset of concepts or skills. & Credit for partially correct answers. \\
Limited ability to assess problem-solving or thought processes. & More time intensive to grade or evaluate. \\
\hline
\end{tabular}

\section{The Department and Student Populations}

TTU is a large university that at the time of this study had about 32,000 students, with 26,000 of them undergraduates. The Physics Department had 20 tenured/tenure-track faculties and taught about 2,600 students (annual enrollment) in the introductory physics courses each year. This included the calculus- and algebrabased introductory physics classes. About 1,800 of these students were in the calculus-based course and 800 in the algebra-based course.

\section{The Laboratories and Recitation Sections}

Prior to Spring 2008, the introductory courses consisted of three-hour of lecture and two-hour of laboratory work each week. The labs were taught by TAs. They were very traditional, "cookbook," in format and pedagogy and had not undergone significant change in many decades. The students would work through the labs and turn in a formal lab write-up to the TA. There was no recitation.

After a transitional semester in Spring 2008, EB laboratories and a one-hour weekly recitation section were introduced into the introductory courses. With this restructuring, the TAs was now trained in different pedagogies than were used in the traditional labs. The traditional lab pedagogy had been random and varied, being left up to the individual instructors of each course, but was usually instructor-centered, "sage-on-the-stage" strategies. Most of the EB labs were designed in a format that required interactive-engagement (IE) during the lab to help guide the students. The EB labs, recitation format, and TA training are described in the first paper and in Appendix I.

\section{The Faculty}

The majority of physics faculty members taught traditionally in a lecture-style format. Very few used an EB pedagogy or IE techniques. They focused primarily on the lecture and had minimal involvement in the recitations and laboratories, which were left to the TAs and lab coordinator(s).

A few instructors interacted with the TAs in lab and recitations, contributing to the training of the TAs, the choice of materials and content to be covered in recitations and the pedagogy to be used in lab and recitation. Most of the instructors, who actively participated in the TA training, were instructors who used EB materials and instructional techniques in the lecture.

The instructors labeled by EB in this paper used EB materials and teaching methods in the lecture. 


\section{The Students}

Calculus-based courses (CBII). Large lecture sections of the calculus-based course. The CBII consisted primarily of engineering and computer science majors. The number of students registered for the second course in the sequence was usually around 400, split among two or three instructors. The instruction was primarily traditional lecture, with a one-hour recitation section and a two-hour lab, as described above and in Appendix I. The labs and recitations were common among the three instructors each semester. Students from each of the lecture instructors were mixed in the labs and recitations.

Honors section. There was one honors section of the calculus-based class that was taken by students in the TTU Honors College and by some of the physics majors. It was usually a small class, consisting of 10-24 students. Sometimes, the honors students took the same laboratories and recitations as the large lecture sections, and sometimes they did not, depending on the instructor. We have included the data that we have for one section of honors students who did take the same laboratories as the students in the large lecture sections and had traditional lecture instruction.

Algebra-based courses (ABII). Large lecture sections of the ABII. The ABII consisted mostly of pre-health science majors, including pre-medical, pre-dental, pre-physical therapy, etc.. The number of students registered each semester in the first course in the sequence was usually around 250-300 and had been around 100-150 in the second course in the sequence at the time this data were taken. Except for the INQ section of the course, the students were divided into two lecture sections taught by two lecture instructors each semester. The instruction was primarily traditional lecture, with a one-hour recitation section and a two-hour lab each week. The labs and recitations were common among the two instructors. Students from each of the lecture instructors were mixed in the labs and recitations.

INQ, laboratory-based section. In addition to the changes being made in the large lecture classes, we wished to assess the INQ course, a laboratory-based, inquiry-based course that was developed with National Science Foundation (NSF) funding about 13 years ago and has been taught as a special section of the algebra-based course every semester since then. It was developed explicitly for health science majors, taking their needs, learning styles, backgrounds, and motivations into account. It is taught without a text in a workshop-physics style (Priscilla, 1999) environment and is an inquiry-based course in the manner of Physics by Inquiry (McDermott \& the Physics Education Group at the University of Washington, 1996), developed by the Physics Education Group at the University of Washington, but at the algebra-based level. The materials were developed by modifying and adapting parts of existing materials designed for other populations and integrating them with new units in our own format, creating a course aimed specifically at health science majors.

The curriculum was designed to be taught in a laboratory-based environment with no lecture and no text. However, a text can be used. Students work through the units in groups, learning to develop both quantitative and qualitative models based on their observations and inferences, and then using the models to make predictions and solve problems. The materials consist of the laboratory units, pretests, readings, and exercises. There are also homework sets, exams, and quizzes. The course covers approximately the same content as is covered in the other sections of the class, but with more of a focus on developing models based on experimentation and developing observational, analytic, and critical thinking skills in order to design experiments and work problems. The students sign up on a first-come and first-serve basis.

The FR pre- and post- tests, while designed for the lecture-based classes, were administered to the students in this section of the ABII, also. We would also like to have done the reverse: Using problems 
designed for the INQ course in the large lecture-based classes are similar to a study previously done by one of the authors (Beth, Suzanne, Eunsook, \& Kelvin, 1994), but this is not feasible as part of this project. It may be possible to do this in the future as part of a smaller study. However, with the move to EB materials in the labs and recitations, the pre- and post- tests were more similar to the type of questions asked in the INQ course, as they were now designed with physics education research (PER) in mind. The difference in the administration was more with the timing of the administration, as the INQ course did not have a fixed lab on one topic with a post-test the following week. The post-tests had to be administered as quizzes within the quiz structure of the INQ course.

\section{Development and Administration of Assessment Instruments}

\section{Development}

The FR pre- and post- tests reported on in this study were written for the large lecture sections of both the algebra- and calculus- based courses by three of the researchers/faculty on the NIH grant. Over the course of the grant, sets of FR pre- and post- tests were developed for both the first and second semester courses in the introductory sequence and for both the ABII and CBII. The questions were designed each week, as each semester progressed, to address specific content and skills taught during a particular week in the labs, recitations, and lecture. We attempted to administer the same problems across pedagogies and across the algebra- and calculus- based sequences whenever possible. There were changes made to both the pre- and posttest problems across semesters in some cases, as the study progressed. However, we were able to administer at least identical significant parts of most problems across multiple semesters. In this paper, we focus on electricity and magnetism ( $\mathrm{E} \& \mathrm{M}$ ) problems consistently administered across different lab and lecture pedagogies in both the algebra- and calculus- based courses over one or more of the semesters.

The pre-tests were designed to assess the pre-knowledge of the students on either the lab or recitation content for that week. They reflected elements that faculty/researchers considered to be relevant at that point in the course. The questions might focus on a conceptual aspect, a laboratory skill, or a mathematical skill, as something that the students should understand after the lecture/laboratory/recitation experience of that week. The quizzes were designed to match one of the pre-tests, allowing a means to assess gain (or change) in a student's understanding or skill level after instruction.

Both the pre-tests and the quizzes were written in FR format and required the students to show their work and explain their reasoning. The rationale was that we could gain information not only on students' conceptual understanding, but also on their laboratory and mathematical skills, as well as their explanatory ability and their thinking skills. It also allowed us to design problems with a range of complexity. As we have a lot of data, we have chosen to present three matching pre- and post- tests from the second semester courses as representative examples of the use of locally written FR questions as part of a large-scale assessment. The problems are given in Appendix II.

\section{Administration}

In both ABII and CBII, a pre-test was administered at the beginning of every lab and a quiz (post-test), was administered bi-weekly in the recitation sections. The pre-tests were collected and scanned for research. Pre-tests were not graded, but were counted towards a participation grade and the participation grade credit was not given if the answer did not appear to be a serious attempt at solving the problem or answering the question. Each week that a quiz was given, the quizzes were scanned and saved for research before being graded by the 
TAs and returned to the students. The grading rubric used by the TAs was not necessarily the content analysis rubric used by the researchers, which is given in Appendix III.

\section{Analysis}

For each matching pre- and post- test, there were two parts to the analysis. We first used a rubric to categorize the answers to each part of a problem based on the extent to which a student had answered correctly. On most problems, we categorized the answers as completely correct (CC), correct choice, partially correct explanation (CP), correct choice, incorrect explanation (CI), or incorrect (I). This allowed us to define a gain, a change in an individual students' understanding from pre-test to post-test, and allowed a quantitative comparison locally across courses and pedagogies. We then qualitatively analyzed the incorrect and partially correct answers for common conceptions, common laboratory, or mathematical skills demonstrated, and evidence of thinking skills used or cognitive structures employed. We discuss first the analysis categories and gain and then the qualitative analysis of incorrect and partially correct answers.

\section{Analysis Categories and Gain}

The scanned pre- and post- tests were first analyzed using an analysis rubric to categorize the answers as $\mathrm{CC}, \mathrm{CP}, \mathrm{CI}$, and I. The data could then be reported as the percentage of students in each category. Each set of data were analyzed by two independent researchers using the rubric in Appendix III. The analysis did not differ on more than $5 \%$ of the students' answers on any of the problems. The matching pre- and post- tests and examples of acceptable correct answers are given in Appendix II. The post-test results by the course math level and pedagogy are presented in Figures 1, 3, and 5.

In addition to recording the pre- and post- test results as the percentage of students in each of the four categories: CC, CP, CI, and I, we also defined a gain, the change in an individual student's answers from pre-test to post-test. The gain is determined by comparing the movement between categories for each student. Given the four categories: CC, CP, CI, and I, if a student moves two steps upward, from I or CI to CC, the student is awarded a gain value of two points. If a student moves one step upwards, from CI or I to CP or CP to $\mathrm{CC}$, the student is given a gain value of one point. If the student moves one step down, $\mathrm{CC}$ to $\mathrm{CP}$ or $\mathrm{CP}$ to $\mathrm{CI}$ or I, the student is given a gain value of -1 point. Similarly, movement from CC to I or CI, is awarded -2 points. The student then has a gain score in the range of -2 to 2 points. In addition, there are two ways the student can have no gain. If the student has I or CI on the pre-test and still has I or CI on the post-test, the student gain is reported as 0 point. However, if the student has $\mathrm{CC}$ on the pre-test and still has $\mathrm{CC}$ on the post-test, the gain is reported as $0+$ point. This student has no gain, but understands the concept or skill being assessed. A student then has a gain score in the range of -2 to 2 points, with the possibility of 0 or $0+$ point.

The gain can then be plotted as six possible categories: (a) complete decrease (CD), gain value -2 points; (b) partial decrease (PD), gain value -1 point; (c) no gain (NG), gain value 0 point; (d) no gain positive (NGP), gain value $0+$ point; (e) partial gain (PG), gain value 1 point; and (f) complete gain (CG), gain value 2 points. The gain results, also by course math level and pedagogy, are presented in Figures 2, 4, and 6.

The gain is a nice way of plotting the transition from pre-test to post-test, as it records the individual student's movement from one category to another. There are three positive categories (CG, PG, and NGP) and three negative categories (CD, PD, and NG) represented in the gain plot. It is useful to look at CG, PG, and NGP to see the percentage of students that have demonstrated an understanding, at least partially, of the skills or content knowledge assessed in a particular problem. 

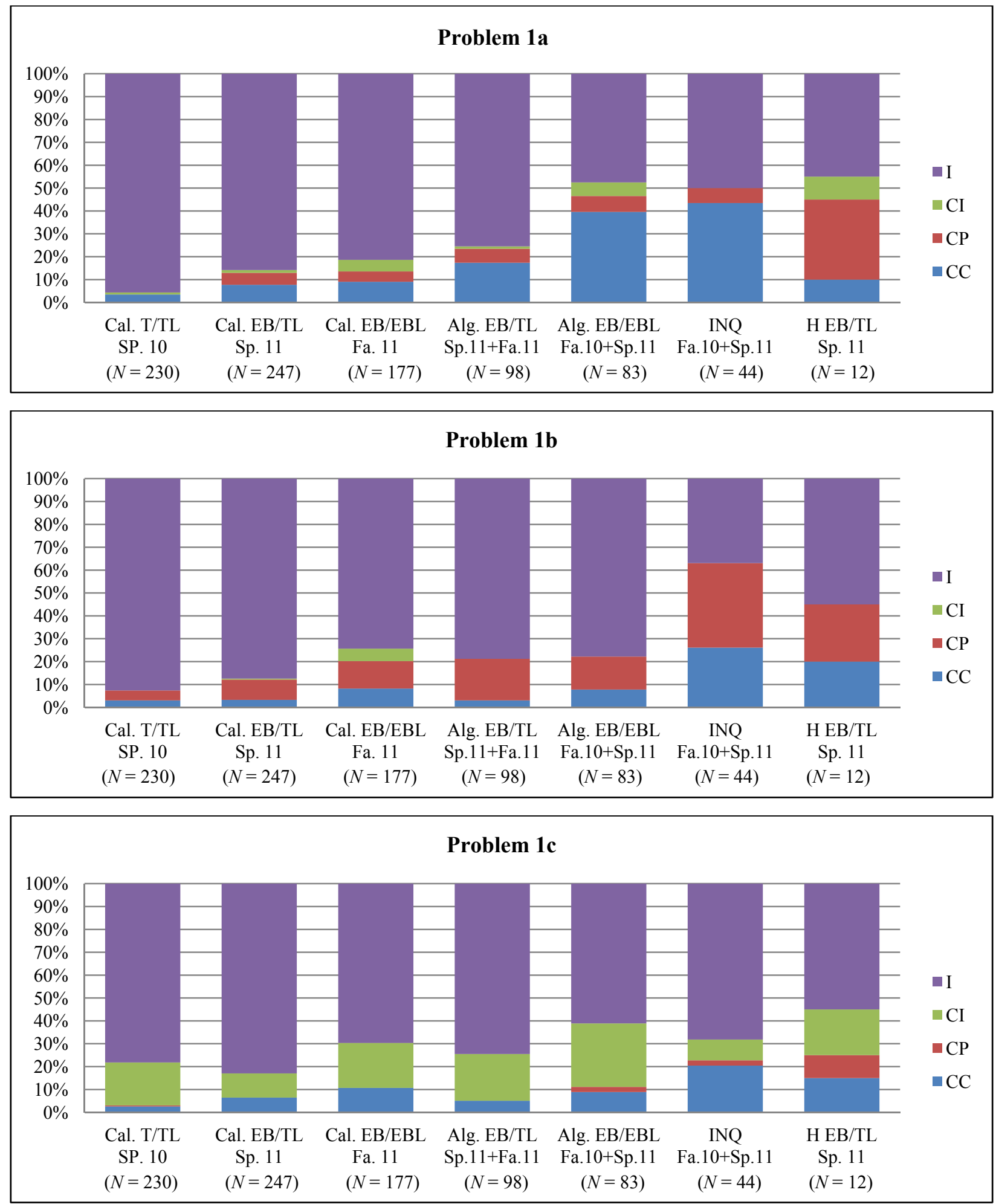

Figure 1. Problem 1 post-test scores across classes, lab, and teaching styles. Answers are categorized as: CC, Correct answer, CP, Correct choice, CI, or I. Laboratory style is categorized by traditional (T) and EB and teaching style is categorized by traditional lecture (TL), EBL, and INQ. Honors sections are indicated with an H. 

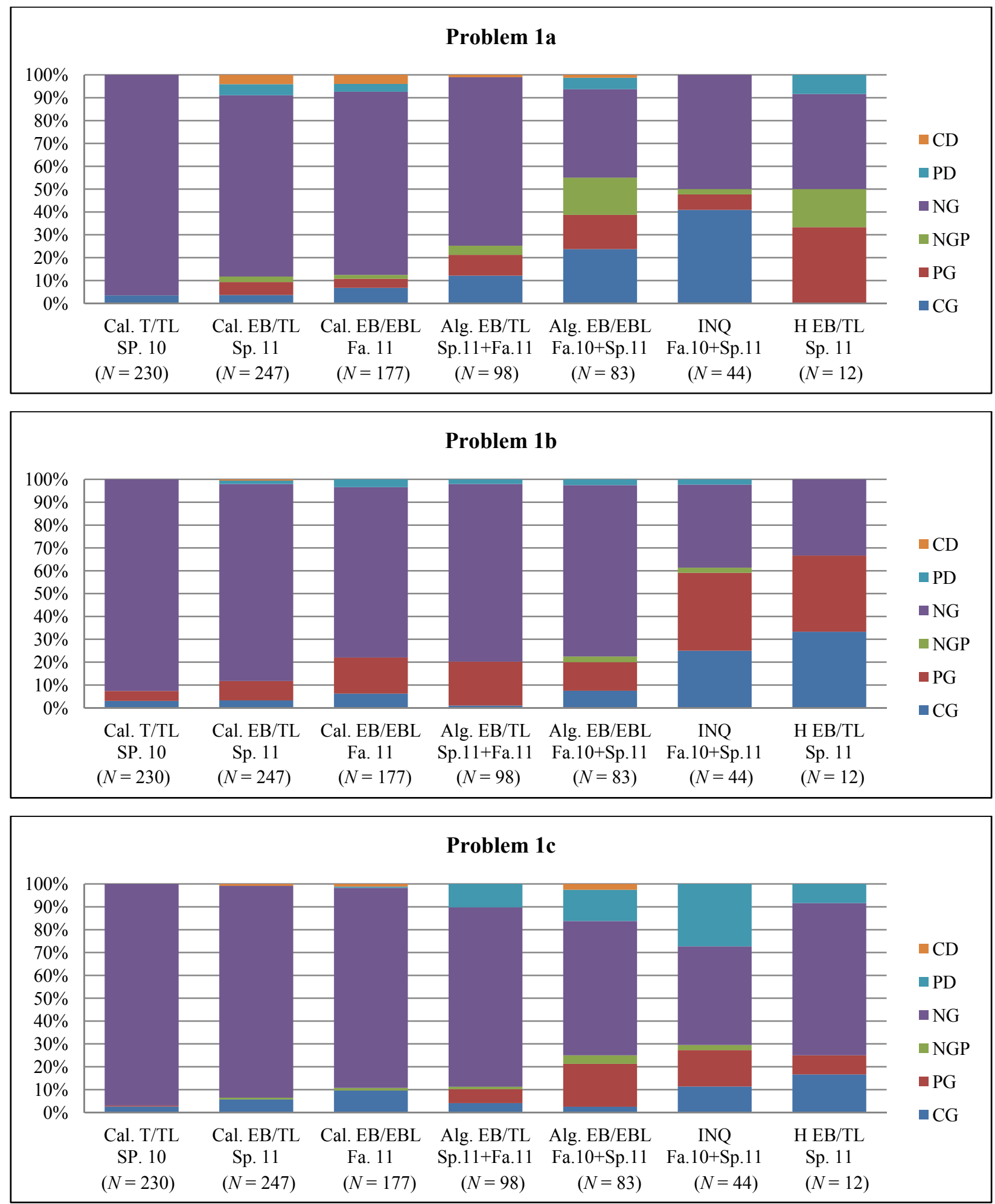

Figure 2. Problem 1 gain values across classes, lab, and teaching styles. Gain is characterized as: CD, PD, NG, NGP, PG, and CG. Laboratory style is categorized by $\mathrm{T}$ and EB and teaching style is categorized by TL, EBL, and INQ. Honors sections are indicated with an $\mathrm{H}$. 

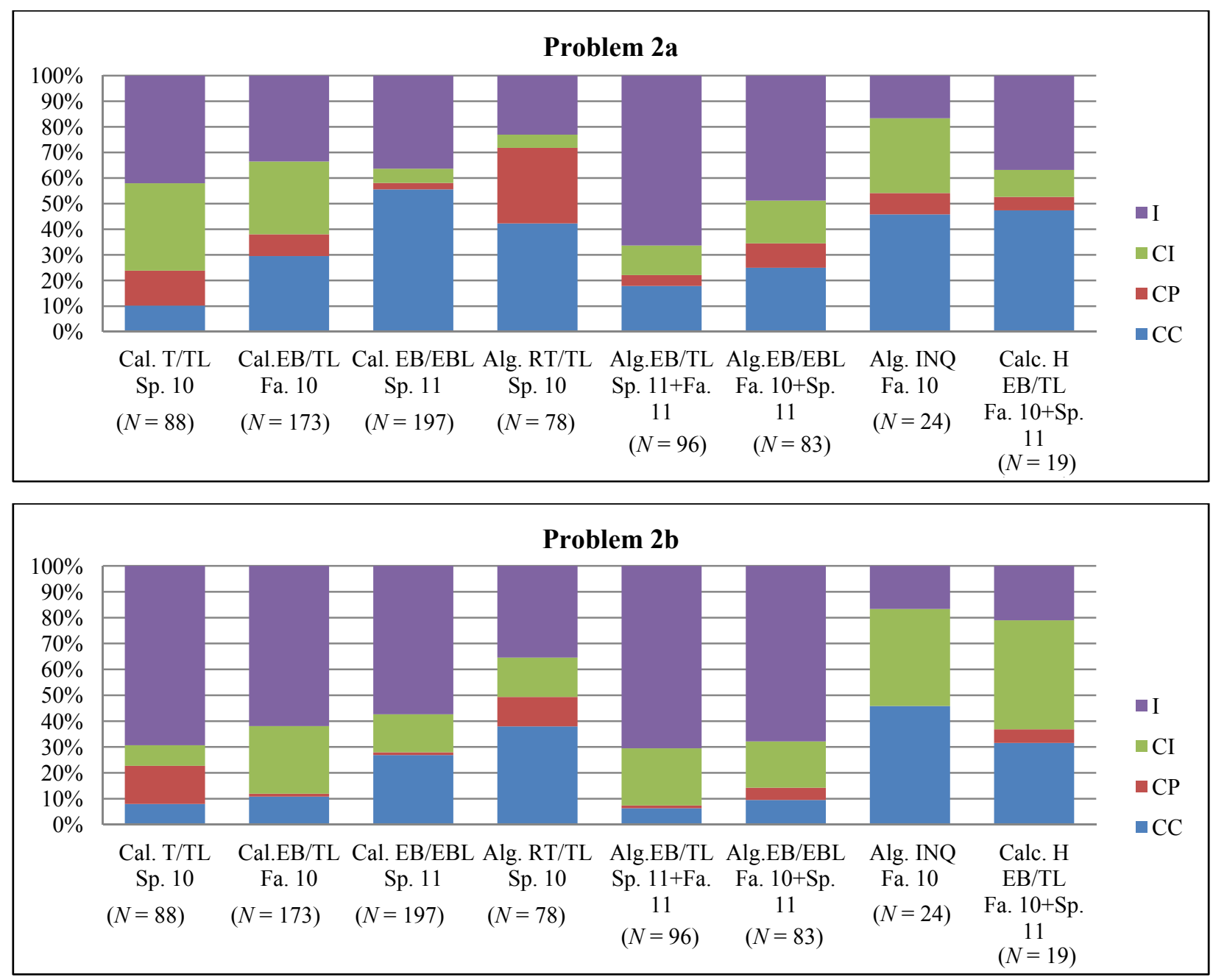

Figure 3. Problem 2 post-test scores across classes, lab, and teaching styles. Answers are categorized as CC, Correct answer, CP, Correct choice, CI, or I. Laboratory style is categorized by $\mathrm{T}$ and EB and teaching style is categorized by TL, EBL, and INQ. Honors sections are indicated with an $\mathrm{H}$.

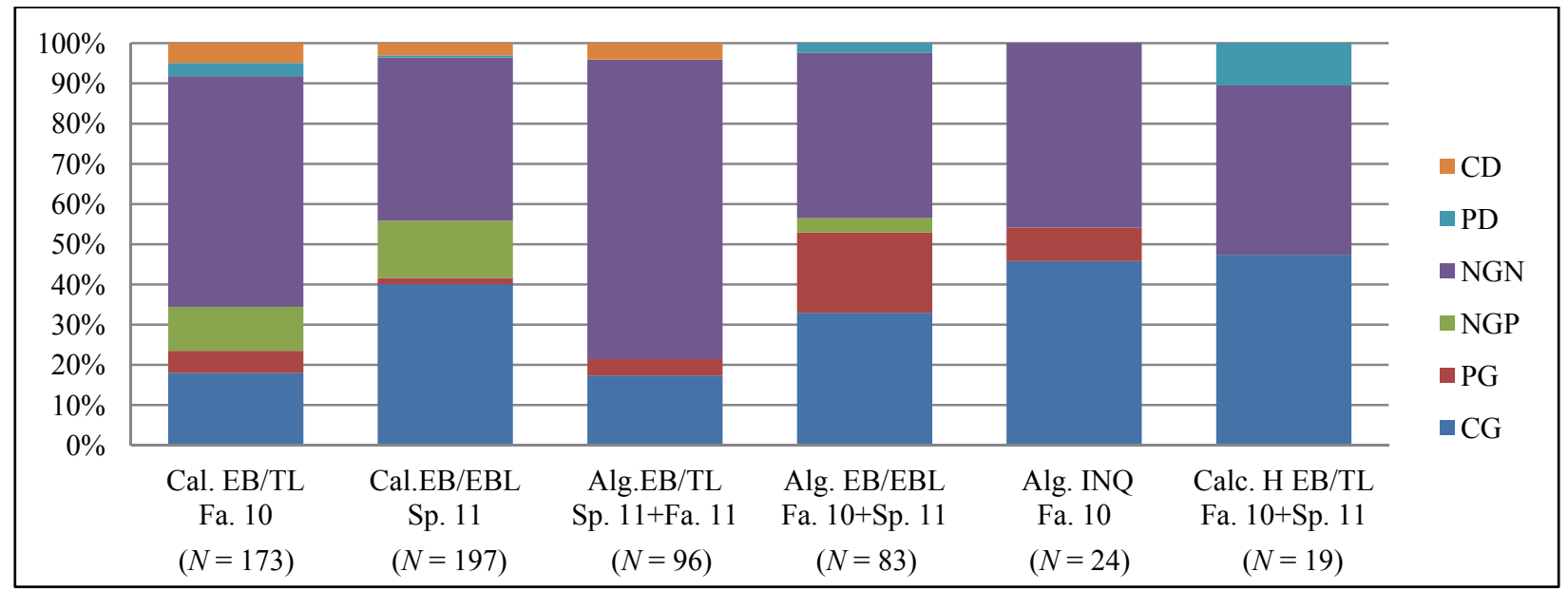

Figure 4. Problem 2a gain values across classes, lab, and teaching styles. Gain is characterized as: CD, PD, NG, NGP, PG, or CG. Laboratory style is categorized by $\mathrm{T}$ and $\mathrm{EB}$ and teaching style is categorized by TL, EBL, and INQ. Honors sections are indicated with an $\mathrm{H}$. 

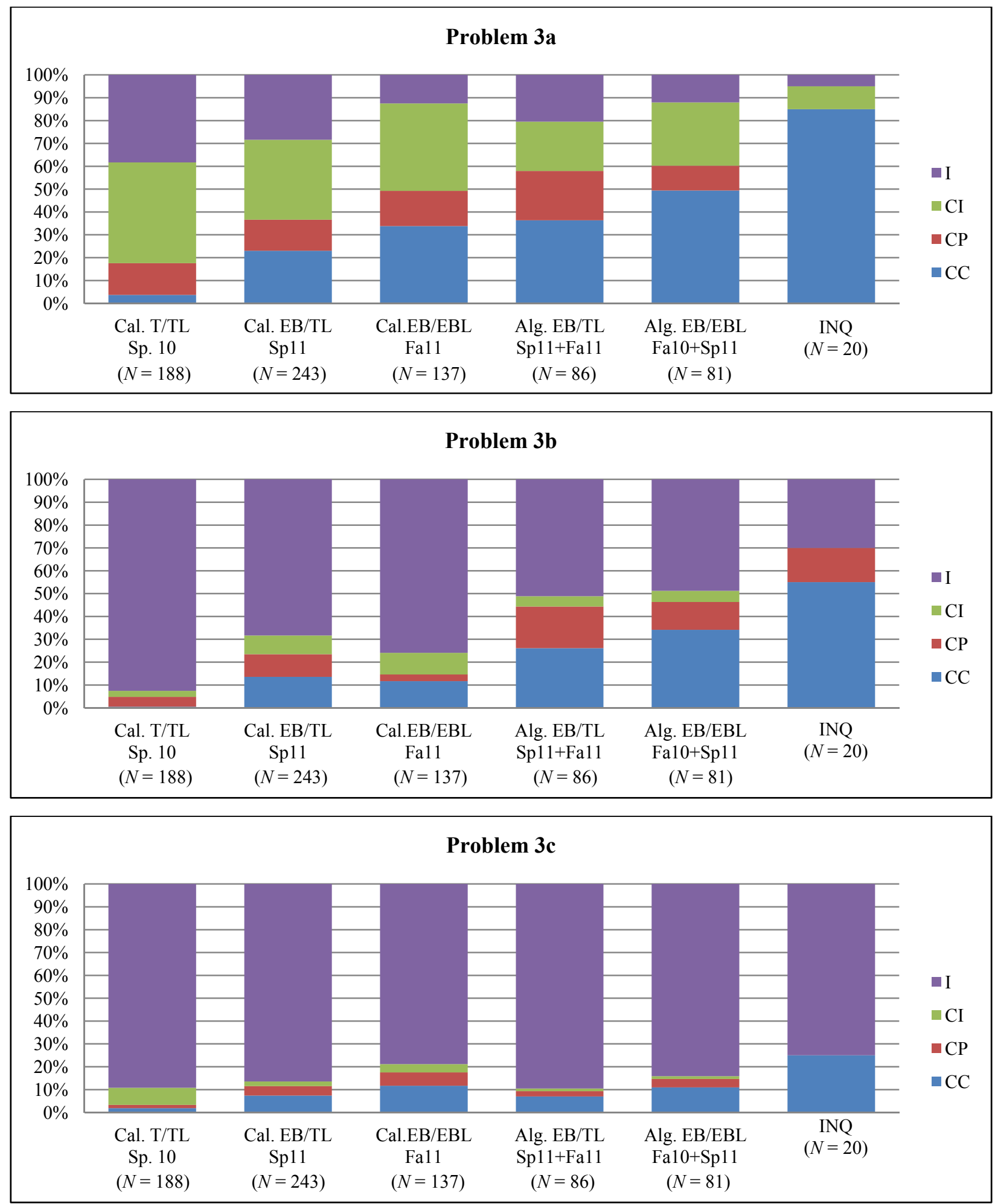

Figure 5. Problem 3 post-test scores across classes, lab, and teaching styles. Answers are categorized as: CC, Correct answer, CP, Correct choice, CI, or I. Laboratory style is categorized by $\mathrm{T}$ and $\mathrm{EB}$ and teaching style is categorized by TL, EBL, and INQ. Honors sections are indicated with an H. 

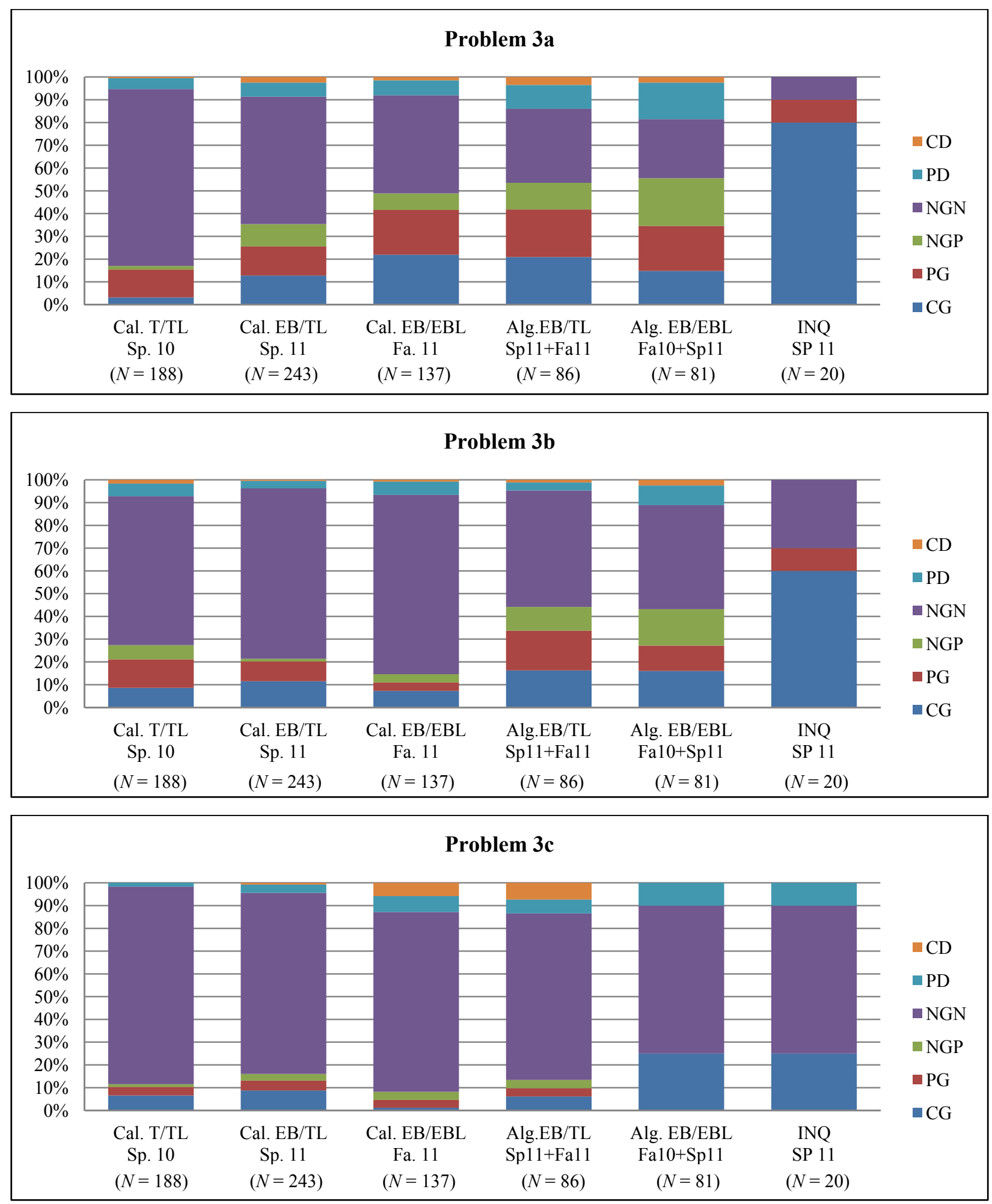

Figure 6. Problem 3 gain values across classes, lab, and teaching styles. Gain is characterized as: CD, PD, NG, NGP, PG, or CG. Laboratory style is categorized by $\mathrm{T}$ and $\mathrm{EB}$ and teaching style is categorized by TL, EBL, and INQ. Honors sections are indicated with an $\mathrm{H}$. 
In the post-test and gain figures, the laboratory and lecture pedagogies were classified as traditional or EB, with the notation T and EB for laboratory and TL or EBL for lecture. The INQ class was simply labeled INQ. The honors section was also categorized by laboratory and lecture pedagogy, separated from the other data, and indicated in the reporting with an $\mathrm{H}$. The course math level is indicated by calculus- or algebra- based. These were the categories useful to the department locally, as it was the introduction of EB materials and pedagogies in the labs and recitations, the use of those pedagogies in the lectures, and the INQ course that we were most interested in assessing. We were interested in the results of individual problems, but also in patterns across problems and across pedagogies and course math level at TTU.

\section{Qualitative Analysis}

As part of the assessment, in addition to categorizing answers based on correct choices and explanations and calculating a gain, we also noted common incorrect and partially correct answers and solution processes and recorded these. This information was useful: (a) for comparison to known pre- and post- conceptions of other students on similar questions reported in the literature; (b) to identify student difficulties related to specific skills, such as mathematical or laboratory skills; (c) to examine the evidence of students' ability (or inability) to explain their reasoning; and (d) to observe evidence of common cognitive resources employed in formulating answers. We were particularly interested in information not available from conceptual inventories. The observations of incorrect and partially correct answers are described below. Also, the samples of common incorrect answers and explanations are given in Appendix IV (Partially correct answers were limited by the rubric as described in Appendix III).

\section{Results}

We give an item by item analysis for the sample problems, pointing out evidence of students' common incorrect conceptions, mathematical difficulties, difficulties with laboratory problems, difficulties explaining their reasoning, and common cognitive resources employed in formulating answers. This is information that, for the most part, is not assessed by conceptual inventories, but reflects strengths and weaknesses of the students in a number of areas that should be addressed as part of programmatic change and change in materials and instructional practice. We also present the local comparisons of post-tests and gain by course math level and pedagogy. This indicates levels of comparative success across the introductory courses, allowing a comparison of skills assessed beyond those assessed by conceptual inventories.

\section{Problem 1 Electrostatics}

The first problem is a problem on two charged parallel plates, given early in the semester when students were studying electrostatics. The problem is given in Appendix II. In between the pre- and post- test, the students in the large lecture sections ABII and CBII had done an experiment in lab that consisted of two 2-D charged conducting plates on conducting paper of essentially the same set-up as in the problem. They experimentally determined the location of equipotential lines and the direction of the electric field based on measurements with a voltmeter and used those measurements to calculate the magnitude of the electric field. The INQ students did not work through that particular lab, but had similar questions as part of thought experiments in their lab. The problem has three parts. The first part is a conceptual question, the second part is a laboratory question, and the third part requires understanding the meaning of an equation.

Post-test and gain analysis. The post-test and gain results are presented in Figures 1 and 2. Figure 1 
presents the post-test and Figure 2 presents the gain results for each part of the problem by course math level and lab and lecture pedagogy. The number $N$ represents the number of students whose matched pre- and posttests were analyzed.

A primary observation of the post-test and gain results are that within both the algebra- and calculusbased classes the percentage of $\mathrm{CC}$ answers rises with the increased use of EB instructional methods in the lectures and laboratories. This was also seen in the conceptual inventory data.

Other observations are that no scores are particularly high, calculus-based classes with traditional labs $\mathrm{T}$ and TL teaching style had the lowest, practically zero, post-test and gain scores, and the INQ students outperform the honors students in all parts of the problem.

The considerable percentage of $\mathrm{CP}$ answers in Part $\mathrm{b}$ is due to a correct explanation that does not explicitly identify the potential difference measurement to be made in the direction of the electric field or between equipotential lines, as explained in Appendix III.

Looking at the CG, PG, and NGP gain categories together, the algebra-based EB/EBL large lecture class, the INQ class, and the honors section are at least at $50 \%$ completely or partially correct understanding on Part a and the INQ class and the honors section are above $60 \%$ completely or partially correct understanding on Part $b$.

Qualitative analysis. Problem 1 Part a. Common incorrect responses to Part a are given in Appendix IV. The most common error was to rank the electric field magnitude by the proximity to one of the plates. Students either ranked the field magnitude as higher closer to the positive plate or higher closer to the negative plate. Over $70 \%$ of the incorrect answers fell into this category. The student answers were not expressed in terms of knowledge of the magnitude and direction of the electric field between two charged plates based on physics content knowledge, such as a calculation or an understanding of an electric field line or equipotential line representation, but were expressed in wording indicative of the use of a cognitive structure known as a phenomenological primitive (p-prim) (Andrea, 1993; John, Smith, Andrea, \& Jeremy, 1993; David, 1996; 2000). In this case, the majority of students seemed be using the p-prim "closer is stronger," a cognitive element that would be appropriate for analyzing the electric field of a point charge, but is not appropriate for analyzing the field between the plates of a capacitor. The high percentage of this type of incorrect answer indicates a need for pedagogy and materials to help students restructure their cognitive resources, helping them to move from the use of more naïve resources towards a more productive structuring of resources. It is clear that the EB materials in the algebra-based EB/EBL classes and the INQ class are more successful at doing this. The EB/EBL methods were less successful in the calculus-based classes on this problem.

Problem 1 Part b. The incorrect answers in Part b were characterized by vagueness, lack of detail and incompleteness. The students answering incorrectly did not demonstrate the ability to identify and verbalize the key measurements they had made in the previous week's lab and write a clear and concise description of the measurements and how to calculate the magnitude of the electric field. The INQ and honors students performed better than the large class sections. From just the answers to this single problem, it is not possible to determine why many students answered vaguely with a lack of detail (not possible to distinguish laziness or inability to explain from an incorrect understanding of the concepts, for example). However, it is clear that many students did not demonstrate an ability to identify and verbalize the key concepts learned and measurements made in the previous week's lab and use that knowledge to answer questions in a clear and concise manner (examples of this are given in Appendix IV). From this problem, we learned that many of the students either were not able to or did not see the need to demonstrate their laboratory knowledge through a clear and concise explanation. 
Problem 1 Part $c$. The most common incorrect answer to Part $\mathrm{c}$ was to choose (ii) and argue that " $E=$ $\Delta V / d$ " was the equation learned in class, indicating evidence of the ability to memorize or recognize an equation, but not the ability to correctly apply it. There was not evidence of a correct understanding of the meaning of the symbols in the equation.

\section{Problem 2 Electric Circuits}

Pre- and post- tests on electric circuits varied considerably throughout our study. We report two parts of a problem that we have post-test data for across our introductory courses. We report the gain on one of those problems, as that is the only electric circuit problem gain that we have across all course math levels and lab and lecture pedagogies. We report the gain only for classes that we have matching pre- and post- test data on that problem. The post-test data are presented in Figure 3 and the gain data are presented in Figure 4. The gain data matches Pre-test Problem $2 \mathrm{~b}$ to Post-test Problem 2a.

On this problem, we also have data from one semester in the large lecture section ABII course that used mostly material from Real Time Physics (David, Sokoloff, Ronald, Thornton, \& Priscilla, 2004) in the laboratories. While Real Time Physics is also EB material, we label it RT and separate it out from locally written EB materials.

Post-test and gain analysis. Students, in general, did better on this problem than on Problem 1. Four groups of students: the INQ class, the calculus-based class with EB labs and EBL instruction, the algebra-based class with RT labs and TL instruction, and the honors class all had CC scores between $40 \%$ and $60 \%$ on Part a. The INQ class and the algebra-based class with RT labs and TL instruction also had scores roughly in that range on Part b. Again, the INQ class outperformed all of the other classes on the post-test, including the honors students, and was roughly equal to the honors students in gain.

This problem was given later in the semester than Problem 1 and is the one problem where the calculus-based students with the same pedagogy outperformed the algebra-based students. It is interesting that it occurred on an electric circuit's problem, as the calculus-based students are predominantly engineering students and may have been familiar with circuits before they took the course.

The gain is given for those classes for which we have matching pre- and post- tests. It reflects the same trends, with the INQ class, the calculus-based EB/EBL class, and the honors class, in this case, with the most gain. The calculus-based courses have a higher percentage of NGP, indicating more correct knowledge of the content before the pre-test was taken.

Qualitative Analysis. Problem 2 Part a. Common incorrect responses are given in Appendix IV. The most common incorrect answer in Part a was to argue that the total resistance increases when a resistor is added and that the current through the battery therefore decreases. The next most common argument was that the current remains constant, independent of the number of resistors. Both of these conceptions have been well documented and reported in the literature (Shaffer \& McDermott, 1992).

If one focused on the cognitive structure, one observes a few things, such as: (1) students answering CC were much more likely to include a very clear and concise logical argument, demonstrating not just their knowledge structure, but their ability to give a clear, explanatory answer; and (2) students answering incorrectly usually just gave an incorrect statement, which, in some cases, reflected an incorrect conception, such as the examples in Appendix IV. However, some students' incorrect answers demonstrated that they were trying to structure an argument from what they believed to be relevant cognitive resources. They were demonstrating thinking skills even though the answer was incorrect. 
It became apparent to us that the solutions to these and other FR problems can be studied more rigorously than in our present project for evidence and analysis of students' use of higher level thinking skills. We have decided to examine the same data more rigorously with a rubric based on Bloom's taxonomy to study thinking skills. We will publish these results in a later paper.

Problem 2 Part b. The most common incorrect answer in Part b was that the voltage across resistor A remains the same, because it is independent of the other resistors connected. This response has also been well documented (Shaffer \& McDermott, 1992).

The same observations on cognitive structure were made as in Part a of this problem and it is interesting to note the percentage of students that chose the correct answer, but were not able to correctly explain their reasoning. This was common in a number of problems in our study, not just in the examples given in this paper and we have begun a further study on this issue.

\section{Problem 3 Induction}

Problem 3 assesses the students' understanding and ability to use Faraday's and Lenz's Law. Between the pre- and post- test, the students in the large lecture sections had worked through a laboratory in which they had performed both qualitative experiments demonstrating Faraday's and Lenz's Law experiments in which they used measurements of a changing magnetic field and the current through a solenoid to calculate the average electromotive force through the solenoid two different ways. The INQ class had done qualitative experiments that demonstrated Faraday's and Lenz's Law and studied the mathematical use of the equations to solve problems. The post-test and gain graphs are given in Figures 5 and 6 . The gain is given for those classes for which we have both the pre- and post- test data.

Post-test and gain analysis. Here again, the INQ students outperformed the students in the other sections, the algebra-based students outperformed the calculus-based students and the traditionally taught students with traditional labs perform the least well. This problem was administered near the end of the semester. The INQ students had a high percentage of CC answers on this problem, with about $75 \%$ completely correct on Part a and about $50 \%$ completely correct on Part $\mathrm{b}$. The algebra-based classes and the EB/EBL calculus-based class had between $30 \%$ and $50 \% \mathrm{CC}$ answers on Part a. The algebra-based classes were also above $20 \% \mathrm{CC}$ on part $\mathrm{b}$.

The gain scores reflect the same trend as the post-test scores. The algebra-based classes have a higher percentage of NGP, indicating that they already knew the concept before the pre-test.

Qualitative analysis. Common incorrect answers are given in Appendix IV. Many of the incorrect answers in Part a reflected a conception that the loop simply had to be in or partially in the field, but not that the flux had to be changing for there to be a current. Some students thought the location of the ammeter determined whether current flowed, i.e., if the ammeter was inside the field, there would be current.

In Part b, many students used the right hand rule incorrectly. Some students did not understand that the external magnetic field was not produced by the currents in the loops.

In Part c, the answers varied widely. There were not common incorrect answers. In this part of the problem, in particular, there were very few attempts to base their answers on the physics of the problem, the relationship between the electromotive force and the current, and the electromotive force and the change in flux. Students were more likely to make some kind of statement about the surface features of the problem, based on the areas of the loops, or the area of each loop inside the magnetic field, with no attempt to even mention how the current through a loop might be related to a particular surface feature. 


\section{Discussion and Conclusions}

We have presented sample data illustrating the use of FR pre- and post- testing to gain information beyond that assessed by conceptual inventories as part of a large-scale assessment. We found that locally written FR problems when systematically administered across courses with different math levels and taught with different pedagogies in the lectures, labs, and recitations yielded information on students' mathematical and laboratory skills, explanatory ability, and cognitive resources, as well as their conceptual understanding that was not available through assessment by conceptual inventories. The results served as a second form of assessment, different from and complementary to conceptual inventory assessment for the comparison of different pedagogical approaches in the lectures, labs, and recitations of our introductory courses. The two forms of assessment were used together to create a much broader picture of our students understanding and skills that could be used to inform faculty and administrators about our students' understanding and learning skills and as the basis for continued development and re-design of instructional materials and pedagogical approaches.

The use of FR pre- and post- testing gave us a much more comprehensive analysis of our students' abilities and skills, identifying deficiencies that would have gone unnoticed if our assessment had consisted of conceptual inventories only. Based on our total study, not just the examples in this article, we have evidence that many of our students: (1) do not demonstrate an ability to clearly explain their reasoning; (2) are often using naïve cognitive resources to structure their arguments; (3) have weaknesses in their understanding of mathematical equations; and (4) are weak at explaining or employing skills and concepts learned in the laboratory. These results are useful for course and program improvement, as we can make changes based on evidence of common weaknesses in mathematical and laboratory skills, the lack of explanatory ability exhibited, and the examples of common structuring of cognitive resources. We can modify our materials and pedagogy in an effort to improve our instruction.

In addition, while we have reported on incorrect answers and weaknesses, which indicate areas that need improvement, one could equally well report on the students who answered completely and partially correctly, elucidating the skills they have mastered, such as the ability to explain their reasoning, correct use of equations, an understanding of concepts and skills learned in lab, and an effective application of their cognitive resources. While we have not written these out explicitly in this paper, elucidating success at these skills is often important for course and program assessment and institutional accreditation. This analysis will be included in a subsequent paper on thinking skills using on a rubric based on Bloom's taxonomy (Bloom, Engelhart, Furst, Hill, \& Krathwohl, 1956; Anderson et al., 2001) that is in progress.

Also, the fact that INQ students performed better than all of the other populations on almost all of the FR questions is of interest. These are questions that yield more evidence of mathematical and laboratory skills, explanatory ability, and thinking skills. While the conceptual inventories are valid and reliable instruments and the FR questions, at the present time, are locally developed, this does suggest that the INQ course is more successful at developing skills other than conceptual understanding and at least equally effective at improving students' conceptual understanding. We expect that this would also be true for other non-traditional, EB curricula and suggest that this type of assessment would be useful at demonstrating the effectiveness of some of these curricula at teaching skills beyond conceptual understanding.

The results also indicate areas for further research and future pathways for assessment. In particular, the qualitative results suggest that more quantitative research could be done on students' thinking skills as 
evidenced by FR physics problems and we are designing a rubric for this purpose. We also suggest that, while we have demonstrated the effectiveness and the use of locally written FR problems for the assessment of skills beyond conceptual inventories, it should be possible to generate a database of vetted, validated, and reliable FR problems (with rubrics) that could be used for comparisons not just locally, but across institutions. We are also working on this.

In summary, we have reported a study on the use of locally written FR problems in identifying students' abilities and skills not assessed by conceptual inventories. The results of such a study can be used as part of cyclical course and curriculum development, program and institutional assessment and a comparison of instructional materials and pedagogies that goes beyond conceptual inventories.

\section{References}

Adams, W. K., Perkins, K. K., Podolefsky, N. S., Dubson, M., Finkelstein, N. D., \& Wieman, C. E. (2006). New instrument for measuring student beliefs about physics and learning physics: The Colorado learning attitudes about science survey. Phys. Rev. ST Phys. Educ. Res., 2, 010101.

Anderson, L. W., Krathwohl, D. R., Airasian, P. W., Cruikshank, K. A., Mayer, R. E., Pintrich, P. R., ... Wittrock, M. C. (2001). A taxonomy for learning, teaching, and assessing: a revision of Bloom's taxonomy of educational objectives. New York, $\mathrm{N}$. Y.: Pearson, Allyn \& Bacon.

Andrea, A. D. (1993). Toward an epistemology of physics. Cognition and Instruction, 10, 105.

Beth, T., Hani, D., Dave, P., \& Keith, W. (2014). Lessons from a large-scale assessment: Results from conceptual inventories. Phys. Rev. ST Phys. Educ. Res., 10, 020104.

Beth, T., Suzanne, M. L., Eunsook, K., \& Kelvin, T. (1994). Comparing problem-solving performance of physics students in inquiry-based and traditional physics courses. Am. J. Phys., 62(7), 627-633.

Bloom, B. S., Engelhart, M. D., Furst, E. J., Hill, W. H., \& Krathwohl, D. R. (1956). Taxonomy of educational objectives, handbook I: The cognitive domain. New York, N. Y.: David McKay Co Inc..

David, M. C., Halloun, I., \& Hestenes, D. (1985). The initial knowledge state of college physics students. Am. J. Phys., 53, 1043-1055.

David, H. (1996). Misconceptions or p-prims: How may alternative perspectives of cognitive structure influence instructional perceptions and intentions? Journal of the Learning Sciences, 5(2), 97-127.

David, H. (2000). Student resources for learning introductory physics. American Journal of Physics, 68 , S52.

Ding, L., Chabay, R., Sherwood, B., \& Beichner, R. (2006). Evaluating an electricity and magnetism assessment tool: Brief electricity and magnetism assessment. Phys. Rev. ST Phys. Educ. Res., 2, 010105.

DiSessa, A. A. (1993). Toward an epistemology of physics. Cognition and Instruction, 10, 105.

Halloun, I., \& Hestenes, D. (1985). The initial knowledge state of college physics students. Am. J. Phys., 53, 1043-1055.

Hammer, D. (1996). Misconceptions or p-prims: How may alternative perspectives of cognitive structure influence instructional perceptions and intentions. Journal of the Learning Sciences, 5(2), 97-127.

Hammer, D. (2000). Student resources for learning introductory physics. American Journal of Physics, 68 , S52.

Hestenes, D., \& Wells, M. (1992). A mechanics baseline test. The Physics Teacher, 30, 159-165.

Jennifer, W., Beth, T., \& Ronald, W. (2007). Creating constructivist physics for introductory university classes. Electronic Journal of Science Education, 11(2), 19-37.

John, P., Smith, III., Andrea, A. D., \& Jeremy, R. (1993). Misconceptions reconceived: A constructivist analysis of knowledge in transition. The Journal of the Learning Sciences, 3(2), 115-163.

Lawson, A. E. (1978). The development and validation of a classroom test of formal reasoning. J. Res. Sci. Teach., $15,11$.

MacIsaac, D. L., \& Falconer, K. A. (2002). Reforming physics education via RTOP. The Physics Teacher, 40(8), 479-485.

McDermott, L. C., \& the Physics Education Group at the University of Washington. (1996). Physics by inquiry volumes I and II. New York, N.Y.: John Wiley \& Sons, Inc..

McDermott, L. C., \& Shaffer, P. S. (1992). Research as a guide for curriculum development: An example from introductory electricity. Am. J. Phys., 60, 994. 
Maloney, D., O’Kuma, T., Hieggelke, C., \& Van, H. A. (2001). Surveying students' conceptual knowledge of electricity and magnetism. Am. J. Phys., 69, S12.

Priscilla, W. L. (1999). Workshop physics activity guide. New York, N. Y.: W. John \& Sons, Inc.

Shaffer, P. S., \& McDermott, L. C. (1992). Research as a guide for curriculum development: An example from introductory electricity. Part II: Design of instructional strategies. Am. J. Phys., 60, 1003.

Smith, J. P., diSessa, A. A., \& Roschelle, J. (1993). Misconceptions reconceived: A constructivist analysis of knowledge in transition. The Journal of the Learning Sciences, 3(2), 115-163.

Sokoloff, D. R., Ronald, K., Thornton, \& Priscilla, W. L. (2004). Real time physics active learning laboratories module one (2nd ed.). New York, N. Y.: John Wiley \& Sons, Inc..

Thacker, B., Dulli, H., Pattillo, D, \& West, K. (2014). Lessons from a large-scale assessment: Results from conceptual inventories. Phys. Rev. ST Phys. Educ. Res., 10, 020104.

Thacker, B., Diaz, A., \& Eligon, A. N. (2007). The development of an inquiry-based curriculum specifically for the introductory algebra-based physics course. arXiv: Physics, 0702247.

Thacker, B., Lea, S., Kim, E., \& Trefz, K. (1994). Comparing problem-solving performance of physics students in inquiry-based and traditional physics courses. Am. J. Phys., 62(7), 627-633.

Wilhelm, J., Thacker, B. \& Wilhelm, R. (2007). Creating constructivist physics for introductory university classes. Electronic Journal of Science Education, 11(2), 19-37.

\section{Appendix I}

\section{PER-informed labs, recitation sections, and TA training}

\section{PER-informed labs}

The locally written PER-informed labs were designed by instructors with knowledge of PER literature, other PER-based instructional materials and pedagogical content knowledge (PCK). The labs consisted of five parts: objectives, overview, explorations, investigations, and summary.

(1) The objectives listed the concepts and skills the students should understand and be able to demonstrate after completing the lab.

(2) The overview was a short summary of the purpose of the lab.

(3) The exploration section was the part of the lab designed to address common student difficulties and conceptions by posing appropriate questions to elicit, confront and resolve the difficulties through questioning and discussion with other students and the TAs. The explorations were qualitative measurements, or sometimes, qualitative problems or thought experiments designed to focus on concepts the students often have difficulty with, even after instruction. They were designed to focus on known difficulties and conceptions in an experimental setting, allowing students to make observations that might challenge or contradict their present conceptual understanding, and allow them to reshape their conceptual understanding through thought and discussion. The concepts were also chosen to support or relate to the investigation section of the lab.

(4) The investigation section of the lab consisted of quantitative measurements and observations, taking data, graphing, analyzing, and interpreting it. Care was taken to elicit predictions before data was taken and to guide the data taking, graphing, and analysis, not being overly prescriptive, but allowing student thought input at each step. Still, it is the section that is more like a traditional lab.

(5) In the summary, the students were asked to focus on a particular part of the lab and summarize it in their own words.

There was also lab homework to be completed and turned in at the next lab, but no formal lab report. Examples of locally written PER-informed labs can be found on a TTU curriculum development webpage.

\section{Recitation sections}

The recitation sections were about 50 minutes long and were usually group problem solving sessions monitored by the TA. The problems were chosen by the lab coordinator(s) and were often chosen from or modified versions of published PER-informed 
problems, such as problems from Tasks Inspired by Physics Education Research (TIPER), Ranking Task Exercises in Physics, books by Arnold Arons, and other sources. Sometimes, the problems were textbook problems or modified textbook problems. The problems were chosen to be on content that had already been covered by all of the instructors teaching the course. The problems were chosen to cover concepts or skills that students often struggle with, even after instruction.

The students would work through the problems in groups, working on whiteboards, with the TA circulating, asking students questions, or answering questions from students. After students had had a significant amount of time to work on the problem, the TAs checked on students' understanding in different ways. Some TAs worked with groups individually, checking on their results both as they worked and as they finished, asking them to explain their results, and asking further questions, as needed. Others called the class together and had groups present at the board and had a class discussion about the problems.

If there was time after the problem(s) for that week had been finished, the TAs entertained questions on homework or other questions students might have. The bi-weekly quizzes were also administered during the recitation sessions.

\section{TA training}

The TAs were trained and directed by the lab coordinator(s). Previous to the introduction of the PER-informed materials, the lab format was very traditional. The lab coordinator(s) were one of the instructors teaching a lecture section of the course. They met with the TAs weekly to "go over" the lab, which may or may not have included actually working through the lab. TAs would start the lab by giving a short presentation or summary of the lab, including important aspects of the experiment and the results that needed to be recorded and reported, as well as the expected format of the lab report. There was usually not a discussion of pedagogy or student conceptions and the instructional format was pretty much left up to the TAs. Lab reports were either turned in at the end of the lab or the next week, depending on the instructor. The TA training and lab format was quite varied and dependent on the instructor serving as lab coordinator in each course. There was no recitation section.

With the introduction of PER-informed labs, it was necessary to train the TAs in student-centered pedagogy. The lab TAs would spend time at their weekly meeting working in groups through the laboratory they would be teaching the following week, with the lab coordinator(s) modeling the teaching methods to be used by the TAs. In the exploration parts of the lab, in particular, the TAs were taught to guide the students through questioning and discussion, eliciting and challenging the students conceptions and helping them to restructure their conceptions.

In the recitation sections, the TAs would spend time each week working in groups through the recitation materials they would be teaching the following week, with the recitation instructor circulating, asking questions, and checking on their understanding. The instructors modeled the pedagogy to be used by the TAs. The TAs was taught to guide the students through questioning, not "telling" answers, but helping students to think through the questions themselves. They were taught how to help each group and to make sure everyone in the group contributed and were responsible for their own understanding. They were also taught how to guide whole class sessions, having groups or students present at the board and then lead class discussions. The use of the IE methods was expected of them both in the recitations and in the exploration parts of the laboratories. The TA's also received training in PCK in a Physics Pedagogy course taken by all first semester graduate students with TA assignments. They learned about PCK through reading PER literature on PCK and working through and discussing student difficulties on particular problems and pedagogical methods for teaching research-based physics problems.

\section{Appendix I}

\section{Problem 1}

1. Pre-test

Consider two parallel charged conducting plates, as in the diagram below. The top plate has a net negative charge and the bottom plate has a net positive charge of the same magnitude. The points $A, B, C$, and $D$ represent points in space (not charges). 


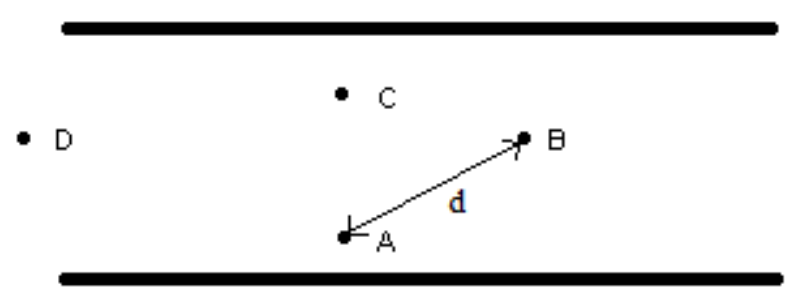

Figure 1. Picture for problem 1 pre-test.

Rank the magnitude of the electric field at each point. Explain your ranking.

How would you experimentally determine the electric field at point $A$ ? Explain.

If the electric field at point $A$ has a magnitude $\mathrm{E}$, the potential difference between points $A$ and $B$ is $\Delta V$ and the distance between points $A$ and $B$ is $d$ (as indicated in the diagram), which of the following statements is true? Circle your choice and explain your reasoning.

$$
\begin{aligned}
& E>\Delta V / d \\
& E=\Delta V / d \\
& E<\Delta V / d
\end{aligned}
$$

2. Post-test with a possible correct answer

Consider two parallel charged conducting plates, as in the diagram below. The top plate has a net negative charge and the bottom plate has a net positive charge of the same magnitude. The points $A, B, C$, and $D$ represent points in space (not charges).

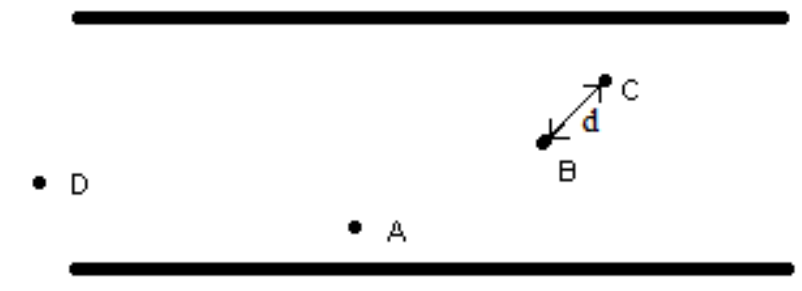

Figure 2. Picture for problem 1 post-test.

Rank the magnitude of the electric field at each point. Explain your ranking.

For two large, charged conducting plates, the magnitude of the electric field is constant between the plates, except at the edges where the field is weaker, as there is a fringe field that bends outward and the field lines are less dense. The field lines point from the positive to the negative plate and are equally dense between the plates. The ranking is " $A=B=C>D$ ".

How would you experimentally determine the electric field at point B? Explain.

Measure the potential difference between two points on either side of point $B$, in the direction of the electric field. The magnitude of the electric field can be found by dividing the potential difference between these two points by the distance between them. The field points directly upwards from the positive to the negative plate. Since the field is uniform between the plates, it is sufficient to measure the potential difference between any two equipotential lines and divide by the distance between them.

If the electric field at point $B$ has a magnitude $\mathrm{E}$, the potential difference between points $B$ and $C$ is $\Delta V$ and the distance between points $B$ and $C$ is d (as indicated in the diagram), which of the following statements is true about the magnitude of the electric field? Circle your choice and explain your reasoning.

$$
\begin{aligned}
& E>\Delta V / d \\
& E=\Delta V / d \\
& E<\Delta V / d
\end{aligned}
$$


(i) $E>\Delta V / d$. The relevant distance to use in determining $\mathrm{E}$ is the distance between the equipotential lines through $B$ and $C$, which is $\Delta x=d \cos \theta$, where $\theta$ is the angle between the electric field and a vector d pointing from $B$ to $C . E=\Delta V / d \cos \theta$, and since $d \cos \theta<d, E>\Delta V / d$.

\section{Problem 2}

1. Part of a pre-test common among classes

Consider the circuit shown in the diagram (1a) below with two resistors in series. A third resistor is added to the circuit, as in diagram (1b).

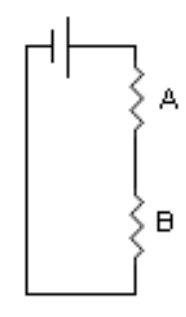

Fig. 1a

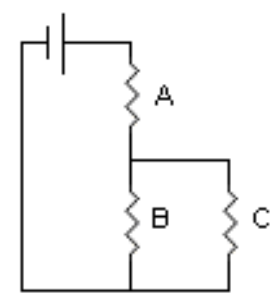

Fig. 1b

Figure 3. Picture for problem 2 pre-test.

Does the total resistance of the circuit increase, decrease, or remain the same? Check the appropriate answer and explain your reasoning.

increases decreases remains the same

Does the current through resistor A increase, decrease, or remain the same after adding resistor $C$ ? Check the appropriate answer and explain your reasoning. increases decreases remains the same

2. Part of a post-test common among classes with possible correct answers

Consider the circuit shown in the diagram (1a) below with two resistors in series. A third resistor is added to the circuit, as in diagram (1b).

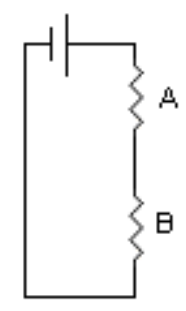

Fig. 1a

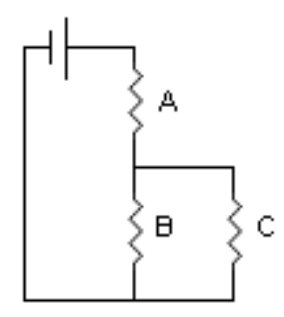

Fig. 1b

Figure 4. Picture for problem 2 post-test.

Does the current through the battery increase, decrease, or remain the same? Check the appropriate answer and explain your reasoning.

$\mathrm{X}$ increase decrease remain the same

When resistor $C$ is added in parallel, the total resistance of the circuit decreases. This can be seen mathematically by solving for the resistance of the $B$-C network, $R_{B C}$, using $1 / R_{B C}=1 / R_{B}+1 / R_{C}$. Then, $R_{B C}=R_{B} R_{C} /\left(R_{B}+R_{C}\right)$ and $R_{B C}<R_{B}$. The total resistance decreases from $R_{\text {Total }}=R_{A}+R_{B}$ to $R_{\text {Total }}^{\prime}=R_{A}+R_{B C}$. When the total resistance of the circuit decreases, the current 
through the circuit increases by Ohm's Law, $V=I R$, with $V$ the battery voltage and $R$ the total resistance of the circuit.

Does the voltage across resistor $A$ increase, decrease, or remain the same? Check the appropriate answer and explain your reasoning.

X___ increase____ decrease___ remain the same

Increases. The total current through the circuit passes through resistor $A$. Since that current increases, the current through $A$ increases. Using Ohm's Law applied to resistor $A, V_{A}=I_{A} R_{A}$, with $R_{A}$ constant, an increase in $I_{A}$, increases the voltage across $A$, $V_{A}$.

\section{Problem 3}

\section{Pre-test}

Consider a loop of wire moving to the right with a constant velocity " $V$ " through a region of magnetic field out of the page, as in the diagram below. The ammeter " $A$ " measures the amount of current flowing through the wire.

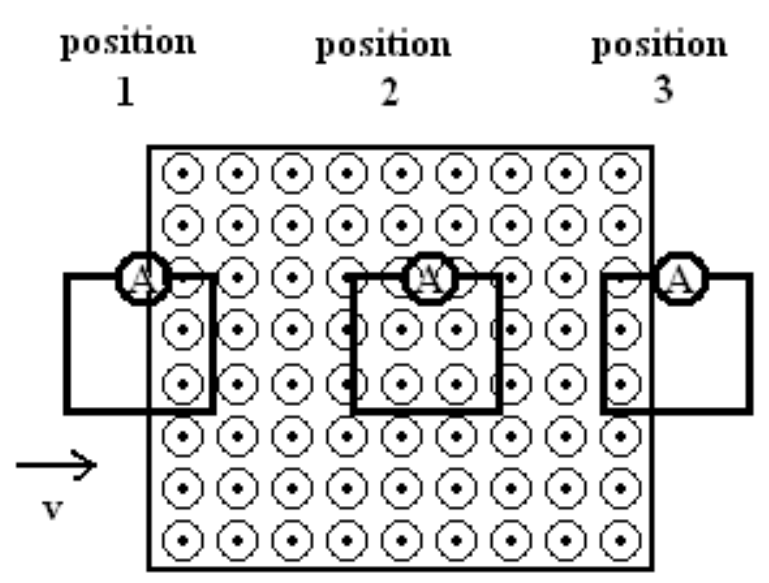

Figure 5. Picture for problem 4 pre-test.

In which case(s), position 1,2 , or 3 , is there current flowing through the wire? Check off the case(s) in which current is flowing through the wire and explain your reasoning.

position 1 position 2 position 3

For each of the cases in Part a in which you indicated that current was flowing through the wire, draw an arrow in the diagram to indicate the direction of the current. Explain your reasoning.

Rank any currents in order from greatest to least. Explain why you ranked them the way you did.

2. Post-test with possible correct answers

(1) Consider three wire loops moving with a constant velocity, $v$, through a region of magnetic field out of the page, as in the diagram below. The wire loops in positions 1 and 2 are moving to the right. The wire loop in position 3 is moving upwards, towards the top of the page. An ammeter " $A$ ", in each loop measures the amount of current flowing through the wire. 


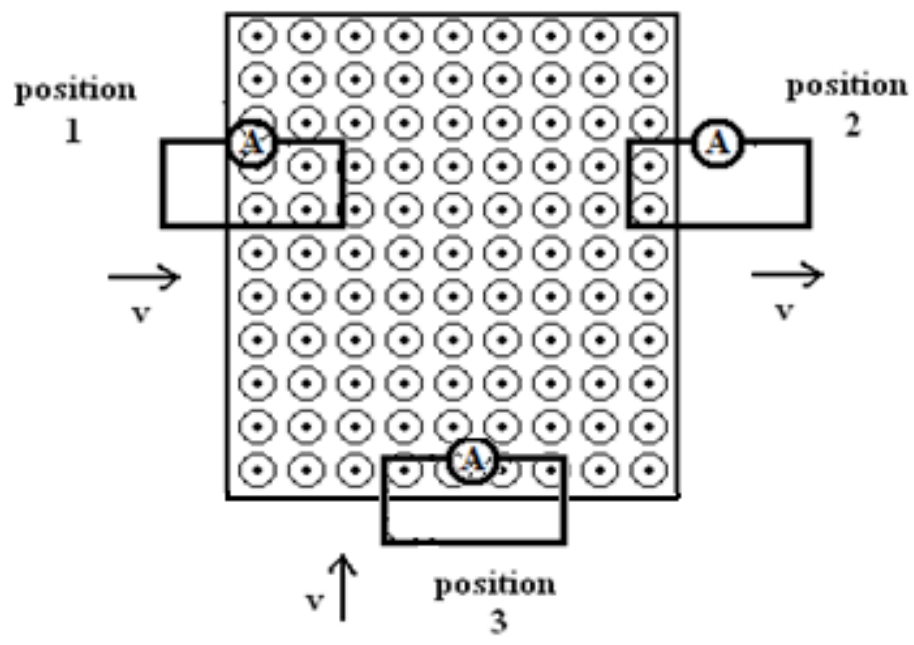

Figure 6. Picture for problem 4 post-test.

(2) In which case(s), position 1, 2, or 3, is there current flowing through the wire? Check off the case(s) in which current is flowing through the wire and explain your reasoning.

$\mathrm{X}$ position 1 $\mathrm{X}$ position 2 position 3

Current is flowing through all of the wires, because the flux through each of the loops is changing with time. $\xi=-N d \Phi / d t$.

(3) For each of the cases in Part a in which you indicated that current was flowing through the wire, draw an arrow in the diagram to indicate the direction of the current. Explain your reasoning.

Clockwise in positions 1 and 3 and counterclockwise in position 2. The induced current flows in the direction that will create an induced magnetic field and an induced flux that opposes the change in flux due to the original field and the moving coils.

(4) Rank any currents in order from greatest to least. Explain why you ranked them the way you did.

Assume identical loops. Then $\xi=I R$ and the current is proportional to the $\xi$. The rate of change of flux is the same in positions 1 and 2, so they will have the same magnitude $\xi$, since $\xi=-N d \Phi / d t$. In loop3, flux changes at a greater rate, since more field lines enter the loop per unit time. So, $3>1=2$.

\section{Appendix II}

\section{Research Rubrics}

1. Problem 1

(1) Part a.

Completely correct. To be completely correct, students had to rank the points as $A=B=C>D$, recognize that the electric field is uniform inside the plates and that the field lines are less dense outside the plates. D, lying outside the plates has a weaker field than the other three points.

Partially correct. An answer was counted partially correct, if it was stated that the field was constant between two large charged, conducting plates, and therefore, the electric field at all of the points were equal.

(2) Part b.

Completely correct. To be completely correct, the answer needed to describe the use of a voltmeter and the placement of the probes to measure a potential difference in the direction of the electric field, and then, identify the correct distance to be measured and used in the equation $E=-\Delta V / \Delta x$.

Note: There were students who described various experiments using charged balls or particles (usually not based on available 
introductory lab equipment). However, none of them were completely correct.

Partially correct. Partially correct answers described a voltmeter measurement between two points, but did not explicitly explain that the measurement should be made in the direction of the electric field. For example, a student would describe placing one of the voltmeter leads on one of the plates and the other at point B, recording the voltage and dividing by the distance between the plate and the point, without explicitly stating that the distance measurement should be made parallel to the electric field lines.

(3) Part c.

Completely correct. A completely correct answer chose (i) $E>\Delta V / d$ and correctly described that the magnitude of $\mathrm{E}$ is $E=$ $\Delta V / \Delta x$, where $\Delta \mathrm{x}$ is the distance between equipotential lines and $\Delta x=d \cos \theta$, where $\theta$ is the angle between the electric field and a vector $\mathrm{d}$ pointing from $\mathrm{B}$ to $\mathrm{C}$.

Partially correct. Partially correct answers were usually incomplete answers. For example, the student would state the correct distance needed, but not explain why that would make $E>\Delta V / d$. They assumed that stating the correct distance needed was a sufficient explanation.

\section{Problem 2}

(1) Part a

Completely correct. To be completely correct a student had to demonstrate qualitatively or through calculations that the resistance of the $B-C$ network is less than the resistance of $B$ and that the total resistance decreases. Then they had to correctly apply Ohm's Law, $V=I R$, with $\mathrm{V}$ the battery voltage and $\mathrm{R}$ the total resistance of the circuit.

Partially correct. A student was awarded partial credit for an answer with the correct process, but an algebraic error or an explanation with a minor error in wording or one that lacked sufficient detail.

(2) Part b

Completely correct. To be completely correct a student had to correctly apply Ohm's Law or a combination of Ohm's Law and one of Kirchhoff's Laws (there are a number of correct ways to work the problem). One of the simplest ways to work the problem is to recognize that the current through A increases and demonstrate qualitatively or mathematically through a correct application of Ohm's law applied to resistor $A$, that the voltage across A increases because the current through resistor A increases, but the resistance remains the same.

Partial correct. Answers were counted as partially correct for the use of Ohm's Law with only minor calculation errors or explanations with errors in wording or that lacked sufficient detail.

\section{Problem 3}

(1) Part a

Completely correct. To have a completely correct answer, the student must recognize that the flux through all of the loops is changing and that it is the changing flux that is inducing a current in the loops.

Partially correct. There were incomplete arguments that addressed the area within the magnetic field changing with time, without explicitly mentioning flux, that were considered partially correct.

(2) Part b

Completely correct. For an answer to be considered completely correct, the student had to demonstrate an understanding of the induced current creating an induced magnetic field that generated an induced flux that opposed the change in flux for all three loops.

Partially correct. There were incomplete arguments of the induced field opposing the magnetic field that were counted as partially correct.

(3) Part c

Completely correct. In order to be considered a completely correct answer, the student had to recognize that the flux through 
loop 3 was changing at a faster rate than the flux through the other two loops and that the flux through loops 1 and 2 was changing at the same rate. Correct answers could be expressed in words or equations. $\Delta \Phi=\Delta(B A) / \Delta t$, where $A=x y$. For loops 1 and $2, y$ is constant and $v=\Delta x / \Delta t$, so $\Delta \Phi=B y v$. For loop 3, $x$ is constant and $y$ is changing, resulting in $\Delta \Phi=B x v$.

Partially correct. Most students wrote equations with little or no explanation; as a result there were very few partially correct answers.

\section{Appendix IV}

\section{Common Student Incorrect Responses}

\section{Problem 1}

We present here some of the common student incorrect responses.

(1) Part a

The most common error in part a was to rank the electric field magnitude by the proximity to one of the plates. Students either ranked the field magnitude as higher closer to the positive plate or higher closer to the negative plate. Over $70 \%$ of the incorrect answers fell into this category. A handful of students ranked the field higher in the middle, decreasing in magnitude as you moved closer to either of the plates.

(2) Part b

Part $b$ incorrect answers were characterized by vagueness, lack of detail and incompleteness. The students did not have the ability to identify the key measurements they had made in the previous week's lab and write a clear and concise description of the measurements and how to calculate the magnitude of the electric field. Examples of vague and incomplete answers follow (These are complete answers. Not parts of answers).

Place a voltmeter at point $\mathrm{B}$ and on the positive plate.

By determining the necessary variables and solving for $\mathrm{E}$ using the proper equation.

You would get $\mathrm{d}$, the distance between them and then get the potential difference between the two points and divide.

I would use a voltmeter to measure the voltage at different points. Then, after obtaining the distance d, I would solve for the magnitude.

(3) Part c

The most common incorrect answer in part c was to choose (ii) and argue that $E=\Delta V / d$ was the equation learned in class. Typical explanations were:

The answer is ii. In class and in the homework, we learned that $E=\Delta V / d$.

This $(E=\Delta V / d)$ is the defined operation.

2. Problem 2

(1) Part a

The most common incorrect answer was to argue that the total resistance increases when a resistor is added and that the current through the battery therefore decreases. Some examples are:

Current decreases, because there is more resistance.

The current will get divided and there is more resistance, so it decreases.

The resistors slow the flow of the circuit. The electrical potential is lost with the resistance of a new resistor.

The next most common argument was that the current remains constant, independent of the number of resistors. Some examples are:

(a) The current does not change and it is wired in parallel.

(b) The current through a given circuit is constant throughout with a given battery. 
(c) Current is constant.

(2) Part b

The most common incorrect answer in part b was that the voltage remains the same, because it is independent of the resistors connected.

Examples of this argument are:

Voltage is constant and unaffected. It is current that is affected by resistors. The battery produces a voltage that is independent of resistors in parallel.

The voltage is unaffected by resistors.

3. Problem 3

(1) Part a

Many of the incorrect answers reflected a conception that the loop simply had to be in, or partially in, the field but not that the flux had to be changing for there to be current.

They are all in the magnetic field.

All loops have a portion of their area in the magnetic field, so there is no reason current will flow through one of them and not the other.

Some students thought the location of the ammeter determined whether current flowed, i.e., if the ammeter was inside the field, there would be current.

The ammeter, along with the positioning, allows the current to flow in the loop as they will have induced current by the magnetic field.

Since there is an ammeter, there is current flowing through the wire, having the magnetic field going through the coil to give current.

(2) Part b

Many students used the right hand rule incorrectly. Examples of this are:

With regards to the right hand rule, since the magnetic field is coming out of the page, the current flows to the right.

Clockwise, I attempted to use the right hand rule.

Using the right hand rule, where our thumb is the current, and the mag field points into the page so the current is clockwise.

If the velocity is going to the right, then according to the right hand rule, the current is directing upwards in the same direction as the force.

Some students did not understand that the external magnetic field was not produced by the currents in the loops.

There is a current going counterclockwise which creates magnetic field lines coming out of the paper which counteracts the increase in the magnetic field's magnitude.

(3) Part c

The answers varied widely. There were not common incorrect answers. 